\title{
Design of a Minimum Surface-Effect Three Degree-of-Freedom Micromanipulator
}

\author{
Michael Goldfarb \\ John E. Speich \\ Department of Mechanical Engineering \\ Vanderbilt University \\ Nashville, TN 37235
}

\begin{abstract}
This paper describes the fundamental physical motivations for small-scale minimum surface-effect design, and presents a three degree-of-freedom micromanipulator design that incorporates a minimum surface-effect approach. The primary focus of the design is the split-tube flexure, a unique small-scale revolute joint that exhibits a considerably larger range of motion and significantly better multi-axis revolute joint characteristics than a conventional flexure. The development of this joint enables the implementation of a small-scale spatiallyloaded revolute joint-based manipulator with wellbehaved kinematic characteristics and without the backlash and stick-slip behavior that would otherwise prevent precision control.
\end{abstract}

\section{Introduction}

Generalized manipulation entails control of both position and force based quantities. Effective implementation of such control is largely influenced by the openloop behavior of the manipulator. In particular, the presence of hard nonlinearities, such as backlash and Coulomb friction, results in significant deterioration of position and force control. The study of direct-drive robots, for example, was borne out of the need to implement precision position and force control of robot manipulators for purposes of mechanical interaction. A direct-drive design significantly reduces the amount of backlash and Coulomb friction in the control plant. The elimination of these hard nonlinearities enables effective and accurate position, force, impedance, or admittance control of the robot manipulator.

Due to the physics of scaling, devices that operate on a microscopic scale are influenced by highly nonlinear surface forces to a much greater degree than those of a conventional scale. Consequently, an effective micromanipulator that will enable dexterous manipulation in a microscopic environment cannot simply be fabricated as a scaled-down version of a conventional scale robot.
Instead, successful development of a microrobot capable of accurate and competent force-controlled micromanipulation will necessitate elimination or intelligent minimization of surface force behavior.

\subsection{Scaling}

The types of forces that dominate mechanical dynamic behavior on a microscopic scale are different than those that dictate motion on a conventional scale. As an example, consider small insects which can stand on the surface of still water, supported only by surface tension. The same surface tension is present when humans come into contact with water, but on a human scale the associated forces are typically insignificant. The world in which humans live is governed by the same forces as the world in which these small insects live, but the forces are present in very different proportions. For the purpose of mechanical dynamics, the forces that govern motion can be generalized into two types: body forces, which directly influence the entire volume of an object, and surface forces, which act primarily on the surface area of an object. In mechanical environments, the former are generally regarded as inertial forces, while the latter give rise to friction forces. Conventional scale mechanics are influenced by both inertial and friction forces, though they are typically influenced to a greater extent by the former. Since the ratio of surface area to volume of an object increases with decreasing size, microscopic mechanics are of a significantly different nature. At a microscopic scale (dimensions on the order of microns), the role of inertial forces is considerably attenuated with respect to surface forces, and some postulate that it becomes altogether insignificant [6].

\subsection{Minimum surface-effect design}

The problem with this change in proportions is that interaction dominated by friction (surface) forces is far more difficult to control than that dominated by inertial (body) forces. The inertial forces are fundamentally smooth, while friction forces, especially those present during sliding, are typically highly nonlinear (e.g.: stick- 
slip phenomena) and quite difficult to control. The net effect of surface-force-dominated behavior is severe deterioration of position and/or force control.

\section{Flexure-Based Design}

Conventional scale mechanisms rely on sliding and rolling at a fundamental level. Kinematic linkages, for example, cannot be constructed without revolute joints, which almost universally incorporate roller or journal bearings. On a conventional scale, such designs can provide near frictionless rotation while bearing significant loads. Since surface forces dominate small scale mechanics, however, geometrically similar designs at a microscopic scale would exhibit significant stick-slip behavior. A flexure-based joint, which utilizes deformation as a means of providing movement, is a viable smallscale alternative to the conventional-scale revolute joint. A diagram of a conventional flexure is shown in Figure 1. The basic characteristics of conventional flexure joints have been studied by several researchers $[3,4,5]$. If properly designed, a flexure-based structure can approximate the motion of a complex kinematic linkage with negligible stick-slip friction and no backlash. Additionally, the absence of rolling and sliding surfaces produces a device that is free of lubricants and thus extremely conducive to clean environments. Preliminary investigations by the authors indicate that a flexure-based micromanipulator design exhibits well-behaved, stable position and force control [2]. For purposes of multi-degree-of-freedom manipulator design, however, conventional flexures have several significant deficiencies. One particularly restrictive deficiency is the limited range of motion. Depending on the geometry and material properties, a flexure will begin exhibiting plastic deformation at ranges on the order of five to ten degrees. In contrast, an ideal revolute joint has an infinite range of motion. Another significant problem with conventional flexures is the poor properties exhibited when subjected to multi-axis loading. An ideal revolute joint is infinitely rigid in all directions of loading except about the desired axis of rotation. In contrast, a conventional flexure exhibits a significant stiffness along the desired axis of rotation and significant compliance along all other axes of loading. A flexure-based joint, for example, will exhibit twist-bend buckling when subjected to twisting. The multi-axis behavior of conventional flexure joints results in both kinematic and dynamic problems, especially when utilizing non-collocated control that relies upon kinematic transformations for task-space accuracy.

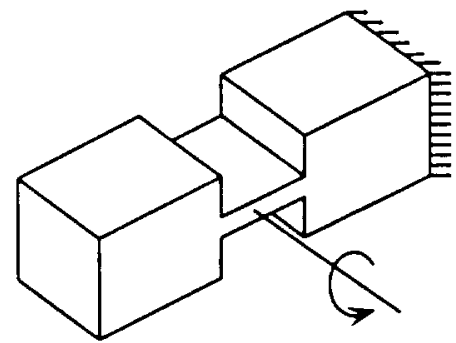

Figure 1. Diagram of a conventional flexure joint, indicating the nominal joint axis of rotation.

\section{The Split-Tube Flexure}

As mentioned previously, an ideal revolute joint is characterized by zero stiffness along the axis of rotation and infinite stiffness along all other axes of loading. Conventional flexure joints offer the benefit of zero backlash and Coulomb friction, but not without limitations. Conventional flexure joints are constrained to a small range of motion and demonstrate significant stiffness along the axis of rotation and significant compliance along other axes. The authors have developed a new flexure that exhibits a considerably larger range of motion and significantly better multi-axis revolute joint characteristics than a conventional flexure. The design of the joint is based upon contrasting the torsional compliance of an open section with its stiffnesses in compression and bending. The torsional mechanics of closed section and open section members are fundamentally and significantly different, while the bending and compressive mechanics of the members are quite similar. This difference in mechanics enables minimization of torsional stiffness and maximization of all other stiffnesses in a nearly decoupled manner.

\subsection{The mechanics of open versus closed sections}

Figure 2 shows two hollow shafts that are in every manner identical except that one has a slit along its long axis. Though geometrically similar, the mechanics of how each bears a torsional load are quite different. For purposes of torsional mechanics, the wholly intact shaft reacts mechanically in the same mode as a solid shaft, while the slitted shaft behaves mechanically as a thin flat plate. This dissimilarity in behavior results in very different torsional stiffnesses. Defining torsional stiffness as the ratio of torque about the long axis to the angular deflection about the same, a stress analysis (assuming 


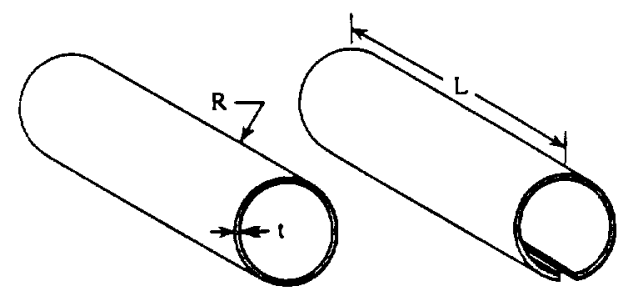

Figure 2. Closed (left) and open (right) section hollow shafts.

typical properties such as linearly elastic, homogeneous, isotropic material) illustrates the differences in torsional mechanics. The torsional stiffness of the closed section, $k_{c s}$, is given by:

$$
k_{c s}=\frac{2 \pi G}{L} R^{3} t
$$

and that of the open section, $k_{o s}$ given by:

$$
k_{o s}=\frac{2 \pi G}{3 L} R t^{3}
$$

where $G$ is the shear modulus of elasticity, and $L, R$, and $t$ are the length, outside radius, and wall thickness of the shaft, respectively. Since by definition, the geometry of a thin-walled shaft is such that $t<R$, the torsional stiffness of the open section is significantly less than of its closed counterpart. For example, a closed-section shaft of radius $R=2.5 \mathrm{~mm}$ and wall thickness $t=0.05 \mathrm{~mm}$ would exhibit a torsional stiffness approximately 7500 times that of a geometrically similar open section.

Though the torsional mechanics of the open and closed section shafts are quite different, the mechanics of bending are in essence the same. Both open and closed section shafts have a bending stiffness given by:

$$
k_{b}=\frac{\pi E}{L} R^{3} t
$$

where $E$ is the modulus of elasticity, and the other variables are as defined previously. Note that this stiffness is quite similar to the torsional stiffness of a closed section shate. In fact, in the limit of $\ll<R$, the joint structure would have a zero torsional stiffness and an infinite bending stiffncss, emulating the ideal revolute joint without the corresponding backlash and Coulomb friction. This, in essence, characterizes the design of the split-tube flexure. Figure 3 shows photographs of a prototype of a split-cube llexure hinge. Note that the axis
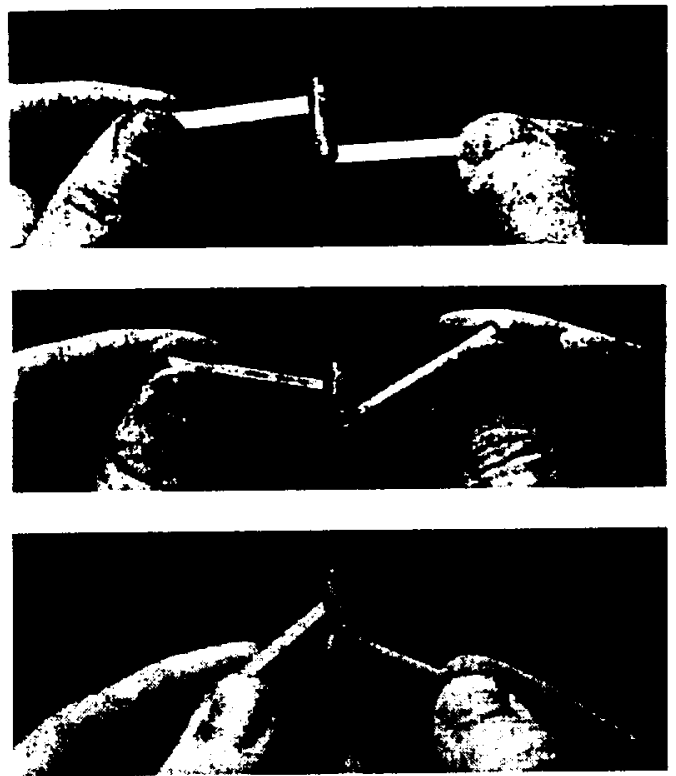

Figure 3. Photographs of split-tube flexure.

of rotation is along the bottom of the tube opposite the split, and not through the center of the tube. Also, though difficult to discern in the photographs of Figure 3, the links adjoined by the flexure hinge remain parallel.

\subsection{The split-tube versus the conventional flexure}

An analytical comparison is useful in assessing the relative mechanical characteristics of both a split-tube and a conventional flexure. Figures 4 and 5 illustrate splittube and conventional flexure geometrys, respectively, and define the loads and deflections from which relevant stiffnesses can be determined. As previously mentioned, the objective in the design of a flexure revolute joint is to achieve a minimal stiffness along the axis of rotation and relatively large stiffnesses along all other axes. The authors refer to the stiffness along the revolute axis as the revolute stiffness, which is defined by $k_{r}=\tau / \theta$. The off-axis stiffnesses of primary interest are the bending stiffnesses, defined by $k_{b 1}=M_{1} / \phi_{1}$ and $k_{b_{2}}=M_{2} / \phi_{2}$ and the axial stiffnesses, defined by $k_{a 1}=F_{1} / x$ and $k_{u z}=F_{2} / y$. Also of primary interest when characterizing joint performance is the allowable range of motion afforded by the joint, a characteristic determined by material yield.

For purposes of comparison, a split-tube and a conventional flexure were designed according to specitications that were determined by a combination of desired manipulator performance characteristics and the 


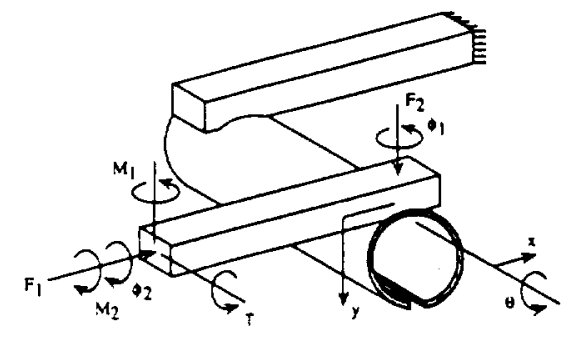

Figure 4. Axes of loading for a split-tube flexure joint.

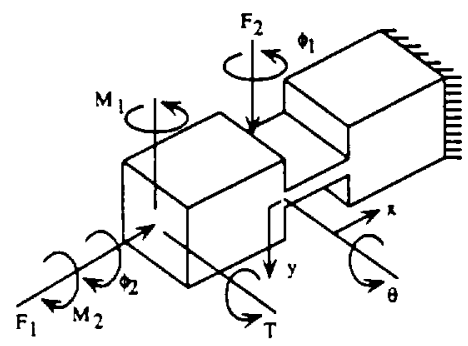

Figure 5. Axes of loading for a conventional flexure joint.

capabilities of the selected actuators. The two joints were designed to have the same revolute stiffness $k_{r}$, and since the micromanipulator is a tendon-based device, the joints were required to have the same axial stiffness $k_{a l}$ and to withstand a given minimum axial load $F_{l}$. Additionally, the two joints were designed of the same stainless steel alloy. The resulting dimensions, as defined in Figures 6 and 7, are $R=2.4 \mathrm{~mm}, t=0.05 \mathrm{~mm}$, and $L=9.5 \mathrm{~mm}$ for the split-tube flexure, and $h=0.2 \mathrm{~mm}, \quad b=0.2 \mathrm{~mm}$, and $l=5.4 \mathrm{~mm}$ for the conventional flexure. The resulting stiffnesses, along with the ranges of motion, are given in Table 1. The characteristics for the split-tube flexure were experimentally verified. Experiments were additionally incorporated to determine maximum load before buckling, a mechanical quantity which is not as well analytically characterized as stiffness or yield. These experiments indicated that the flexures could withstand approximately 8 Newtons before buckling, which is more than 3 times the 2.4 Newton capability of the conventional tlexure. Table 2 incorporates the same information as Table 1, but represented instead as the relative characteristics of the split-tube flexure with respect to the conventional flexure. The ratios of $k_{r}$ and $k_{a l}$ of the two flexures were set equal by design. One can observe from Table 2 that all other off-axis stiffnesses of the split-tube flexure, $k_{h 1}, k_{h 2}$, and $k_{u 2}$ are three to four orders of magnitude larger than the equivalent off-axis stiffnesses of the conventional thexure. Note also that the split-tube flexure enables 150 degrees of motion, more than five

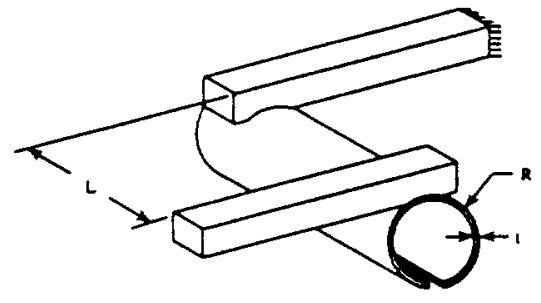

Figure 6. Geometry of a split-rube flexure joint.

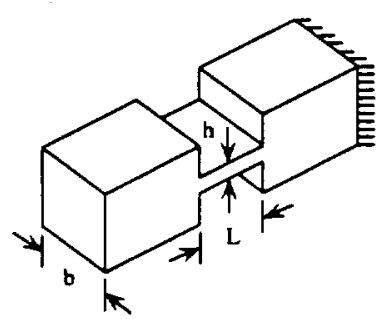

Figure 7. Geometry of a conventional flexure joint.

times that of the equivalent conventional flexure. This comparison clearly illustrates the improved revolute joint properties offered by the split-tube flexure.

Another significant difference between the split-tube and conventional flexure involves the kinematic behavior of the joints. An ideal revolute joint provides a fixed axis of rotation so that the motion of one link with respect to the adjoining link can be characterized as a pure rotation. The illustration of Figure 1 depicts the instantaneous revolute axis associated with a conventional flexure joint. This axis does not remain fixed with respect to either link, but rather translates in the plane as the links rotate. In contrast, the split-tube flexure has a fixed axis of rotation (opposite and parallel to the split), which enables wellcharacterized kinematics with a minimal set of measureients.

\begin{tabular}{|c|c|c|}
\hline Property & $\begin{array}{c}\text { CONVENTIONAL } \\
\text { FLEXURE }\end{array}$ & $\begin{array}{c}\text { SPLiT-TUBE } \\
\text { FLEXURE }\end{array}$ \\
\hline$k_{r}$ & $0.00525 \mathrm{Nm} / \mathrm{rad}$ & $0.00525 \mathrm{Nm} / \mathrm{rad}$ \\
\hline$k_{h 1}$ & $0.00525 \mathrm{Nm} / \mathrm{rad}$ & $43.8 \mathrm{Nm} / \mathrm{rad}$ \\
\hline$k_{h 2}$ & $0.00806 \mathrm{Nm} / \mathrm{rad}$ & $43.8 \mathrm{Nm} / \mathrm{rad}$ \\
\hline$k_{a l}$ & $1.53 \times 10^{6} \mathrm{~N} / \mathrm{m}$ & $1.45 \times 10^{6} \mathrm{~N} / \mathrm{m}$ \\
\hline$k_{u 2}$ & $540 \mathrm{~N} / \mathrm{m}$ & $1.45 \times 10^{6} \mathrm{~N} / \mathrm{m}$ \\
\hline$\theta_{\text {mur }}$ & \pm 14.7 degrees & $\pm 77.9 \mathrm{degrces}$ \\
\hline
\end{tabular}

Table 1. Comparison of conventional and split-tube tlexure revolute joint properties. 


\begin{tabular}{|c|c|}
\hline Property & $\begin{array}{c}\text { Characteristics of SPlit-tube } \\
\text { Relative to ConVentional Flexure }\end{array}$ \\
\hline$k_{r}$ & 1 \\
\hline$k_{h 1}$ & 8330 \\
\hline$k_{h 2}$ & 5440 \\
\hline$k_{a l}$ & 0.95 \\
\hline$k_{a l}$ & 2680 \\
\hline$\theta_{\max }$ & 5.3 \\
\hline
\end{tabular}

Table 2. Relative characteristics of the split-tube flexure with respect to the conventional flexure.

\section{Microrobot Design}

The authors have incorporated the split-tube flexure joints into the design of a three degree-of-freedom revolute micromanipulator. A solid model of the manipulator is shown in Figure 8. The manipulator was designed to be used with a vision system afforded by an optical stereomicroscope, either for human use in a teleoperative sense, or for machine use in an autonomous sense. The workspace of an optical stereomicroscope is approximately $125 \mathrm{~mm}^{3}$ (a cube $5 \mathrm{~mm}$ on a side) and the resolution approximately $1 \mu \mathrm{m}$ (a function of the wavelength of light). The manipulator was designed to have a maximum endpoint force of approximately 100 milliNewtons along each taskspace axis. Since inertial, damping, and elastic forces scale as the geometric scaling factor to the fourth, third, and second powers, respectively, the equivalent elastic (most conservative) force in a workspace cube of $50 \mathrm{~cm}$ a side would be 1000 Newtons. The equivalent forces for geometric similarity in predominately damping or inertial environments would be 100 kiloNewtons and 10 megaNewtons, respectively. Also, since humancontrolled telemanipulation is one of the targeted modes of operation, the manipulator was designed for teleoperative transparency, which generally requires position and force bandwidths on the order of 15 and $300 \mathrm{Hertz}$, respectively [1]. As mentioned previously, one of the primary motives of the micromanipulator design was to eliminate the highly nonlinear stick-slip friction that is imminently present at small scales, in addition to eliminating any hacklash that is exhibited most by sliding and rolling elements. The design philosophy is, in essence, to circumvent intractable control problems by intelligent mechanical design.

The manipulator incorporates a direct-drive semiparallel live-bar linkage revolute configuration. Manipulator actuation is provided by voice coil actuators that intertice with the manipulator via pre-tensioned push-pull rod mechanismss with knife-edge joints. The live-balr actuators (BEI model LAI3-12-000) provide a continuous force of seven Newtons over a stroke of approximately six millimeters. The base actuator (BEI model LA15-33-000) provides a continuous force of sixteen Newtons over a stroke of approximately eleven millimeters. These actuators provide the manipulator with a workspace of approximately $8000 \mathrm{~mm}^{3}$ (a cube $20 \mathrm{~mm}$ on a side) and a maximum endpoint force approaching 500 milliNewtons. The actuators are mounted in flexure suspensions, and thus are devoid of stick-slip friction and backlash. The actuator suspensions include strain-gage-based position sensors for collocated feedback. Note that well-behaved revolute joints are of great import when using sensors in the actuator space, since task-space accuracy is dependent upon well-known kinematic transformations. Force sensing is provided by strain-gages near the manipulator endpoint.

The link lengths of kinematic importance to the manipulator are both $40 \mathrm{~mm}$ long, which enables the manipulator to access the $8000 \mathrm{~mm}^{3}$ workspace with joint ranges of motion of approximately \pm 25 degrees per joint. Figure 9 shows a solid model of the two degree-offreedom five-bar linkage. As shown in the figure, each manipulator revolute joint consists of two split-tube flexures rather than one. The double and single split-tube configurations are referred to as compound and simple configurations, respectively. Arranging the flexures in the compound configuration offers significantly better overail revolute joint behavior. Figure 10 illustrates the compound and simple joint configurations, both of which are constructed of tubes of equal radius and thickness, and both having the same overall length. Mechanical analysis

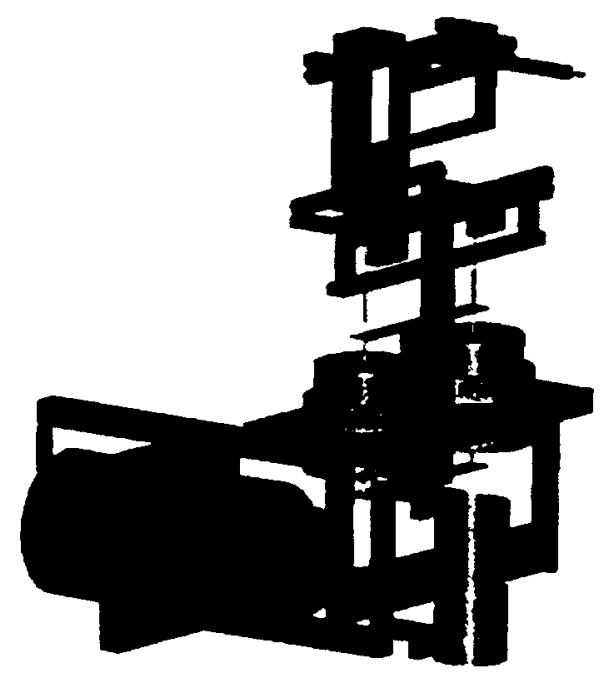

Figure 8. Solid model of split-lube tlexure-hased, voicecoil-actuated three degree-off-freedom micromitnipulator. 


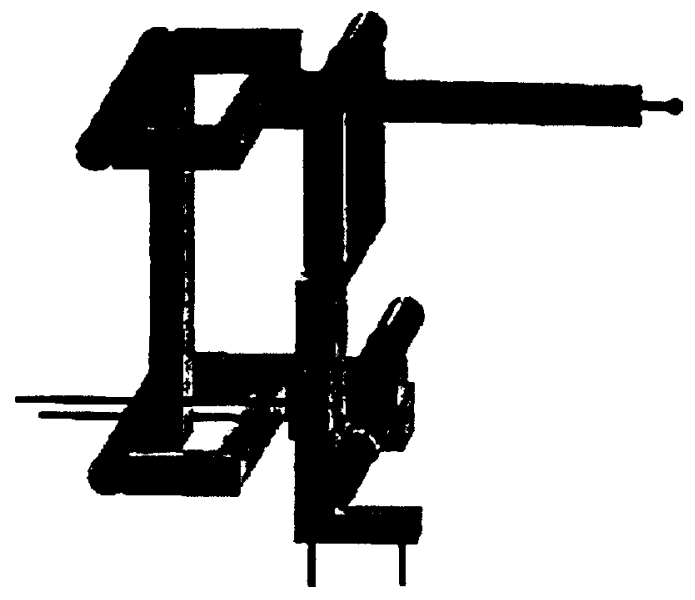

Figure 9. Solid model of two degree-of-freedom five-bar linkage.

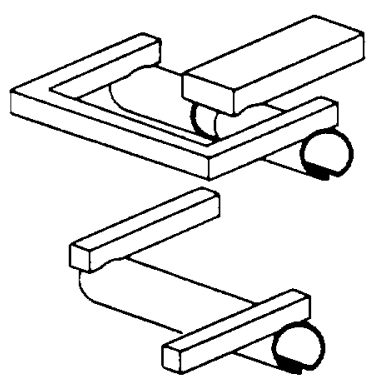

Figure 10. Diagram illustrating the compound (top) and the simple (bottom) split-tube flexure configurations.

shows that for the revolute, axial, and bending stiffnesses defined previously, the compound configuration exhibits 4 times the revolute stiffness, 64 times the axial stiffness, and 16 times the bending stiffness of the simple joint configuration. A flexure-based revolute joint can be characterized by the ratio of revolute stiffness to axial stiffness and the ratio of revolute stiffness to bending stiffness, both of which would approach zero in the ideal case. Though the absolute value of the revolute stiffness is 4 times larger for the compound joint, the ratio of revolute to axial stiffness and of revolute to bending stiffness are 16 and 4 times smaller, respectively, than the simple joint. The compound joint therefore offers better joint behavior, and thus was selected for the micromanipulator configuration.

The micromanipulator is presently under construction. Once functional, the system performance will be characterized and subsequently presented in a future publication.

\section{Conclusion}

The authors have developed a new flexure-based revolute joint that offers significantly better properties than a conventional flexure. The joint:

- exhibits no backlash or stick-slip behavior,

- exhibits off-axis stiffnesses three to four orders of magnitude greater than a comparable conventional flexure,

- enables roughly five times the range of motion of a comparable conventional flexure, and

- withstands roughly three times more load than a conventional flexure.

The development of this joint enables the implementation of a small-scale spatially-loaded revolute joint-based manipulator with well-behaved kinematic characteristics and without the backlash and stick-slip behavior that would otherwise prevent precision control.

\section{Acknowledgments}

Support for this work was provided by NASA Grant No. NAGW-4723. The authors gratefully acknowledge this support.

\section{References}

[1] Fischer P., Daniel R., and Siva K.V., "Specification and Design of Input Devices for Teleoperation," Proceedings of the IEEE Conference on Robotics and Automation, pp. 540-545, 1990.

[2] Goldfarb, M. and Celanovic, N., "Minimum Surface-Effect Microgripper Design for Force-Reflective Telemanipulation of a Microscopic Environment," Proceedings of the ASME International Mechanical Engineering Conference and Exposition, November 1996.

[3] Horie M., Nozaki T.. Ikegami K., and Kobayashi, F., "Design System of Super Elastic Hinges and its Application to Manipulator for Micro-Bonding by Adhesives," Proceedings of the International Symposium on Microsystems, Intelligent Materials, and Robots, pp. 185-188, 1995.

[4] Paros, J. and Weisbord, L., "How to Design Flexure Hinges," Machine Design. Vol. 37, No. 27, pp. 151-156, 1965.

[5] Ragulskis K., Arutunian M., Kochikian A., and Pogosian M., "A Study of Fillet Type Flexure Hinges and Their Optimal Design," Vibration Engineering, pp. 447-452, 1989.

[6] Trimmer, W. and Jebens. R.. "Actuators for Micro Robots." In Proceedings of the IEEE Insernational Conference on Robotics and Automation. pp. 1547-1552, 1989. 